\title{
Assessing microlens quality based on 3D irradiance measurement at the focal spot area
}

Jeremy Béguelin, Michail Symeonidis, Wilfried Noell, Reinhard Voelkel, Toralf Scharf

Jeremy Béguelin, Michail Symeonidis, Wilfried Noell, Reinhard Voelkel, Toralf Scharf, "Assessing microlens quality based on 3D irradiance measurement at the focal spot area," Proc. SPIE 10678, Optical Micro- and Nanometrology VII, 106780B (24 May 2018); doi: 10.1117/12.2306136

SPIE. Event: SPIE Photonics Europe, 2018, Strasbourg, France 


\title{
Assessing microlens quality based on 3D irradiance measurement at the focal spot area
}

\author{
Jeremy Béguelin ${ }^{1}$, Michail Symeonidis ${ }^{2}$, Wilfried Noell ${ }^{1}$, Reinhard Voelkel ${ }^{1}$, and Toralf Scharf ${ }^{2}$ \\ ${ }^{1}$ Süss MicroOptics SA, 2068 Hauterive, Switzerland \\ ${ }^{2}$ Nanophotonics and Metrology Laboratory, École Polytechnique Fédérale de Lausanne,
} 1015 Lausanne, Switzerland

\begin{abstract}
During the fabrication process of microlenses, characterization is essential for two purposes: evaluate the optical quality of the element and provide surface information feedback for process optimization. However, no technique can fulfill these two objectives at the same time. Interferometry is used for quality evaluation and optical profilometry for process optimization. In order to address this problem, we propose to use a high resolution interference microscope to characterize microlenses. The focusing capacity can be directly measured by recording the field near the focal spot at different wavelengths. Information about the microlens surface can also be retrieved. All this is illustrated for the front focus of a fused-silica microlens.
\end{abstract}

Keywords: Micro-optics, Microlens, Interferometry, Aberrations, Spectral characterization, Metrology

\section{INTRODUCTION}

Microlenses and microlens arrays (MLAs) are nowadays widely used for high quality applications. From fiber coupling to custom illumination they are essential optical components of complex devices, such as a Shack-Hartmann sensor or Mask-Aligner [1]. Several techniques exist for their fabrication [2, 3], but all of them require an accurate characterization method in order to optimize the fabrication process and to control their quality.

Surface height measurements are the most important piece of information for a manufacturer in order to optimize the manufacturing process. This explains why optical scanning profilometry, mainly confocal microscopy and coherence scanning interferometry (CSI), is the most widespread characterization technique. However, profilometry works in reflection and consequently, limits the characterization of microlenses in terms of numerical aperture (NA) and field of view (FOV). Moreover, this type of characterization is limited to single microlenses.

On the other hand, microlens quality control is ensured by optical characterization. This generally consists of interferometric measurements, e.g. Mach-Zehnder or Twyman-Green interferometry [4], or in 3D irradiance measurements of the focal spot [5]. These techniques allow the direct determination of the microlens quality by determining wavefront aberration, focal length or Strehl ratio (SR). Working in transmission, these techniques allow the characterization of high NA microlenses and more complex systems.

To combine the advantages of both approaches, we use the spectral High Resolution Interference Microscope (sHRIM) [6] to characterize microlenses. Both interferometric and irradiance measurements can be performed at multiple wavelengths and at different desired positions. This allows the characterization of high NA microlenses when measurements are performed at the focal spot. Moreover, key surface parameters, such as radius of curvature (ROC) and surface deviation, can be obtained from these measurements. Another advantage of this tool is the possibility to characterize more complex systems such as MLAs. That being said, this approach is thus considered to be the most complete characterization technique for microlenses and MLAs.

Further author information: E-mail: jeremy.beguelin@suss.com

Optical Micro- and Nanometrology VII, edited by Christophe Gorecki, Anand Krishna Asundi,

Wolfgang Osten, Proc. of SPIE Vol. 10678, 106780B · (c) 2018 SPIE

CCC code: $0277-786 \mathrm{X} / 18 / \$ 18 \cdot$ doi: $10.1117 / 12.2306136$

Proc. of SPIE Vol. 10678 106780B-1 


\section{METHODS}

The measurements are carried out using the sHRIM, a schematic of which is shown in Fig. 1. In principle, it is a MachZehnder type interferometer that allows the measurement of both amplitude and phase in a certain volume around the sample [7],[8]. In this custom built interferometer, a super-continuum source (SCS) is used for probing the samples properties at different wavelengths.

The output of the SCS is collimated, unpolarised and covers the wavelength range from 480 to $840 \mathrm{~nm}$. The working wavelength is subsequently chosen by an optical variable bandpass filter, before entering the main body of the sHRIM. The first beam splitter (BS) divides the beam in the reference and object arm. The measurement finally determines the phase difference between those arms, pixelwise on the CCD camera used as detector. The second BS recombines the two arms to produce the fringe pattern on the camera. The second output of the interferometer is not used.

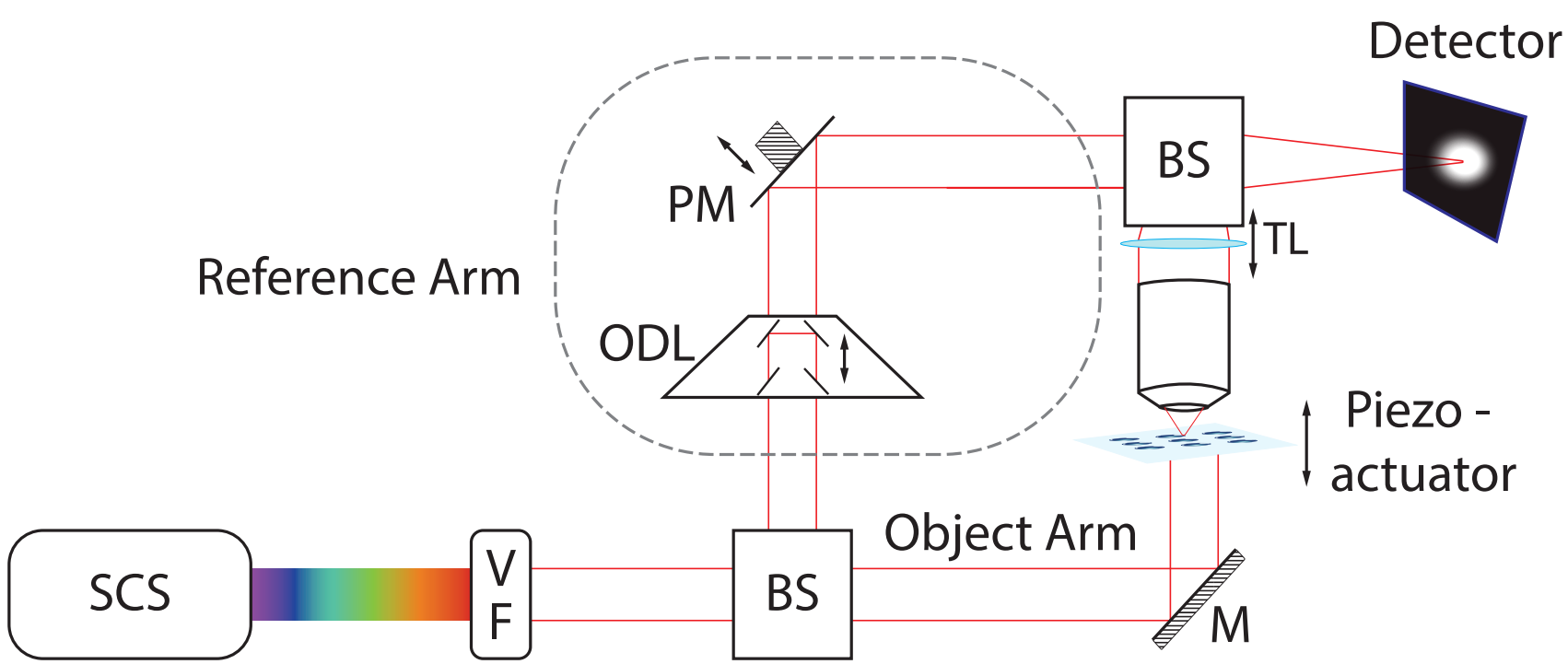

Figure 1: spectral High Resolution Interference Microscope. SCS: Super-Continuum Source, VF: Variable Filter, BS: Beam Splitter, (P)M: (Piezo)Mirror, ODL: Optical Delay Line, TL: Tube Lens. VF selects the working wavelength. The first BS divides the beam, which, after propagating along the different arms, is recombined by the second BS. The interference pattern is recorded by a CCD camera.

\subsection{SHRIM analysis}

The full width at half maximum (FWHM) of the filter output is set to approximately 10nm, which means that the coherence length $L_{c}$ of the beam after the filter is in the order of tenths of micrometers, since $L_{c} \propto \lambda_{0}^{2} / \Delta \lambda$, where $\lambda_{0}$ is the central wavelength [9]. In order to maximize the fringes contrast, the optical path difference between the reference and object arm should be brought close to zero for every wavelength, which is achieved by tuning the (optical) length of the reference arm using the optical delay line (ODL). The stage that operates the ODL has sub-micron step size and a repeatability of few microns, features sufficient for consistent and repeatable results.

Along the beam-path of each arm, different optical components are inserted, which possess different dispersion characteristics. This creates a difference in the optical path length, which is compensated by the ODL, as well as a difference between the radii of curvature of each wavefront. This is evident from the fringe pattern, which consists of concentric rings, if no action is taken. In order to compensate for this effect, the tube lens (TL) is mounted on a translation stage that allows the adjustment of its position. For every wavelength, there is a TL position that restores the straight line fringes (when no sample is inserted in the object arm). It is found that the change in the radius of curvature of the object arm wavefront is negligible over several hundreds of microns of TL movement, which makes the measurement insensitive to position inaccuracies of the TL. It should be noted that the camera and the TL are mounted on the same stage and move in tandem. 
In order to record the phase or the intensity of the light-field emerging from the tested part, a microlens in this case, the sample is mounted on a piezo-actuator and is scanned through the focal plane of the objective. This way the desired quantity, phase or intensity, is mapped on several 2D slices along the axis of propagation. Next, the 3D field of the measured quantity is recreated by combining all the recorded slices. The resolution on the plane perpendicular to the propagation axis depends on the illuminating wavelength, the NA of the objective and the pixel size of the camera. Along the vertical axis, the resolution is set by the step of the piezo stage and is limited by the depth of field of the objective. In general, the resolution on the perpendicular plane and along the propagation axis are different.

Lastly, one mirror is mounted on a piezo stage with a relatively short traveling distance of the order of $10 \mu \mathrm{m}$, but with a step resolution of few nanometers. The stage is operated in closed-loop configuration, which offers great positioning stability and repeatability. This mirror is used to implement the Phase Shifting Interferometry (PSI) [10], which will be explained in the following part.

\subsection{Irradiance and phase measurements}

Measuring the irradiance is straightforward, since blocking the reference arm renders the sHRIM a dia-illumination microscope. Additionally, the ODL and TL position do not affect the measurements. Averaging over five images at each sample stage position is used to reduce the noise.

Phase shifting interferometry is used to capture the phase. Briefly, the total intensity of the interfering fields at each pixel $P(x, y)$ can be expressed as [11]

$$
I_{t o t}(x, y)=I_{d c}(x, y)+I_{a c}(x, y) \cos [\phi(x, y)+\Delta \phi],
$$

where $I_{d c}$ and $I_{a c}$ are proportional to the power of both arms, $\phi(x, y)$ is the phase difference between the two arms and $\Delta \phi$ is an additional phase term that can be changed by translating the piezo mirror. Since $I_{d c}, I_{a c}$ and $\phi$ are unknown, at least three measurements are required to define a system of equations. In this particular implementation we record five different instances, while increasing $\Delta \phi$ by $\pi / 2$ at each successive interferogram. This algorithm is robust and insensitive to deviations from the nominal value of $\Delta \phi[12]$ and the final result is given by:

$$
\phi=\arctan \left[\frac{2\left(I_{t o t, 2}-I_{t o t, 4}\right)}{2 I_{t o t, 3}-I_{t o t, 5}-I_{t o t, 1}}\right] .
$$

The output of the arctan function is limited in the $(-\pi, \pi)$ range and a phase unwrapping algorithm is required to calculate the real (unwrapped) phase difference between the two arms. In this work, the Goldstein algorithm is used to validate that the measurements are residue-free and is compared also to the faster least squares error algorithm [13]. Both algorithms produce equivalent results.

Measurement procedure: The measurements are conducted using the following procedure. Steps in italic are carried out only for the phase measurements.

1. The ODL and TL positions which produce straight fringes without any sample for all wavelengths of interest are found. 2. At each of those wavelengths, setting the TL position according to the values of the previous step, the ODL position which produces fringes when the sample is in the object arm is found. 3. The inclination of the reference arm beam is adjusted, so that a single fringe is apparent on the detector. 4 . The rim of the microlens is brought into focus and the position of the sample stage is recorded. 5. Subsequently, the focal spot of the microlens is brought to focus and the new position of the sample stage is recorded. The difference of the two positions is used to estimate the focal length. 6 . For each wavelength, the phase or irradiance is recorded, at a given range of the piezo-actuator positions.

\section{RESULTS AND DISCUSSION}

As an example, the front focusing capacity of a low NA (0.13) plano-convex microlens is characterized. This spherical microlens made of fused silica is fabricated by photolithography and reactive ion etching (RIE). The microlens surface is also characterized by confocal microscopy to compare the results of both approaches. The surface geometry is defined by its diameter of $240 \mu \mathrm{m}$, by its radius of curvature (ROC) of $424.5 \mu \mathrm{m}$ and by the refractive index of the material, $n \approx 1.45$ in the visible spectrum. For front focal study, the lens thickness is of no importance.

The different measurements are carried out with a 100x objective, NA $=0.95$ and FOV $=63.2 \times 47.6$ microns. This in order to obtain the best lateral resolution for focal spot measurement. 


\subsection{Irradiance measurement}

The $x y$ plan of the 3D irradiance measurement under plane wave illumination is shown in Fig. 2. The pattern exhibits a good symmetry around the z-axis as well as around the focal plane. Qualitatively, this means the level of aberrations is low and the system is almost diffraction-limited.

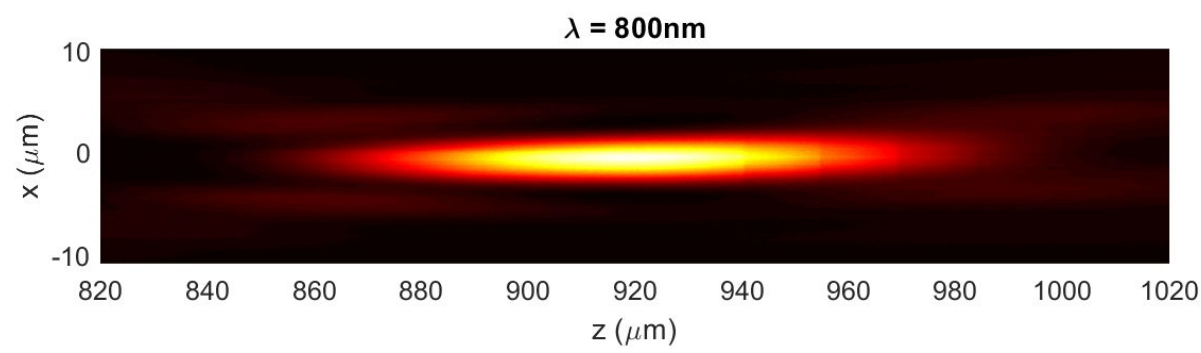

Figure 2: Intensity distribution at the front focal spot of a spherical microlens, $\lambda=800 \mathrm{~nm}$. The origin of the z-axis is set to be the flat outer rim of the microlens.

\subsubsection{Focal length and Strehl ratio}

More quantitatively, selected parts of the entire distribution can provide important information about the focusing capacity. For instance, the intensity on the z-axis, Fig. 3, provides the focal length as well as the depth of focus (DOF). The focal length is defined as the $\mathrm{z}$ position of the maximum intensity. In the ideal case, it is related to the microlens ROC $R$ by

$$
f=R /(n-1)-h,
$$

where $h$ is the lens height. In the present case, the surface measurement predicts a focal length of $919.7 \mu \mathrm{m}$ which is in good agreement with the $918 \mu \mathrm{m}$ determined by irradiance measurement. The DOF is defined in the present case as the width of the intensity peak at $80 \%$ of its maximum [14]. The measured value of $46 \mu \mathrm{m}$ is consistent with the simulated one, $48 \mu \mathrm{m}$.

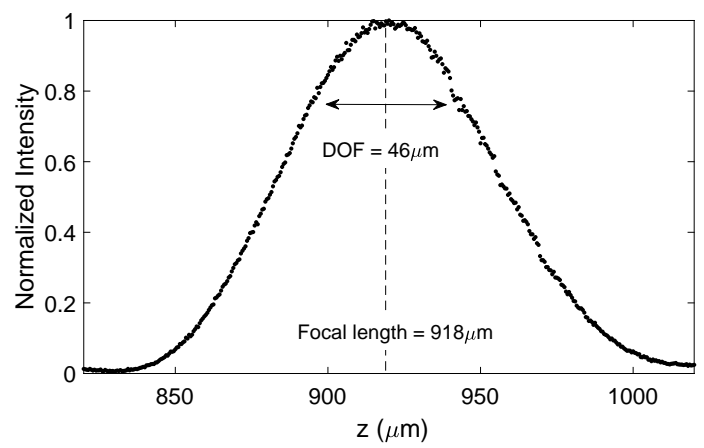

Figure 3: Measured intensity on the optical axis, $\lambda=800 \mathrm{~nm}$. Focal length and DOF can be calculated as the $\mathrm{z}$ position of the maximum intensity, respectively as the peak width at $80 \%$ of its maximum.

\subsubsection{Strehl ratio}

The most common figure of merit to judge the quality of an optical system is the Strehl ratio (SR), which is the ratio between the maximal intensities of real and ideal systems [14]. It represents the amount of aberration present in a real system and is given by the relation

$$
S R=\frac{\max \left(I^{\text {real }}\right)}{I_{\text {ideal }}(0,0, f)}=\frac{\max \left(I^{\text {real }}\right)}{P_{0} \pi \frac{a^{2}}{\lambda^{2} f^{2}}} .
$$


The ideal intensity is given by the far-field diffraction pattern of a circular aperture: with $a$ the microlens radius, $P_{0}$ the total optical power and $f$ the focal length. Practically, $P_{0}$ is the total power at the focal plane recorded on the camera. A correct removal of the ground noise is necessary since it can significantly influence the final result. Fig. 4 shows the real and ideal intensity profiles. For this microlens, the SR takes the value of 0.86 . This microlens is thus diffraction limited according to the Maréchal criterion [14].

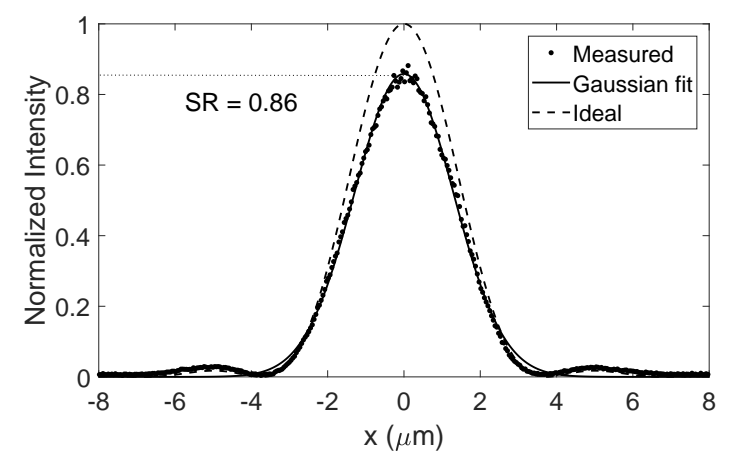

Figure 4: Comparison between ideal and measured intensity in the focal plane, $\lambda=800 \mathrm{~nm}$. The SR is taken to be the maximum of the Gaussian fit.

\subsubsection{Chromatic behaviour}

Fused silica is a dispersive medium and the focusing properties of the tested microlens thus depend on the wavelength. To illustrate this effect, the focal length and the SR are remeasured for two other wavelengths, 500 and $650 \mathrm{~nm}$, then compared, Fig. 5 and 6. Values of the refractive index are 1.4623, 1.4565, 1.4533 for $\lambda=500,650$ and 800nm respectively [15].

Equation 3 predicts a shift of about $20 \mu \mathrm{m}$ of the focal spot when the wavelength changes from 500 to $800 \mathrm{~nm}$. This shift can be observed in Fig. 5b and 6. Inversely, the microlens ROC can be deduced from these measurements using simulations. Indeed, Eq. 3 is not accurate enough due to focal shift induced by diffraction [16].

The SR for the different wavelengths is shown in Fig. 5a. The SR is an increasing function of the wavelength. Indeed, the SR can be written [14]

$$
S R \approx e^{-\frac{4 \pi^{2}}{\lambda^{2}} n^{2} \Delta z^{2}}
$$

with $\Delta z$ the rms value of the surface deviation from the ideal shape. This shows that the SR converges towards 1 for increasing wavelengths. With a surface deviation of $32 \mathrm{~nm}$ measured with the confocal microscope, SRs of approximately $0.71,0.82$ and 0.88 for $\lambda=500,650$ and $800 \mathrm{~nm}$ are expected. This fits well with the measurement of $0.74,0.82$ and 0.86 .

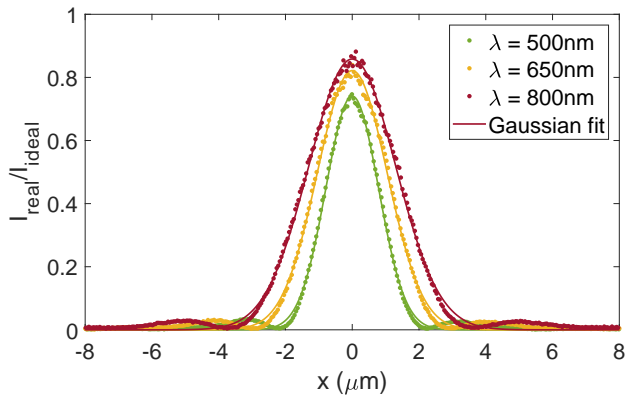

(a)

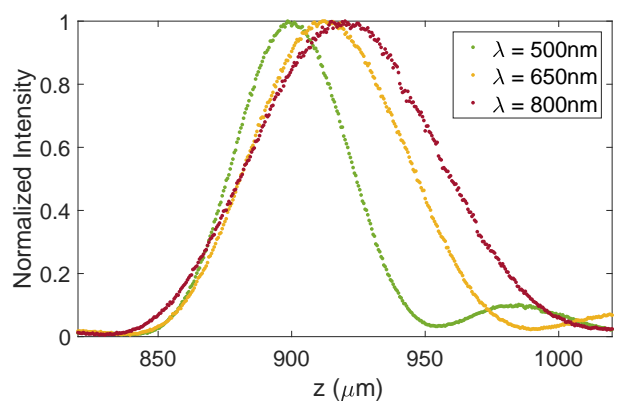

(b)

Figure 5: Measured intensity in the focal plane (a) and on axis (b) for three different wavelengths: $\lambda=500,650,800 \mathrm{~nm}$. The origin of the z-axis is set to be the flat outer rim of the microlens. 

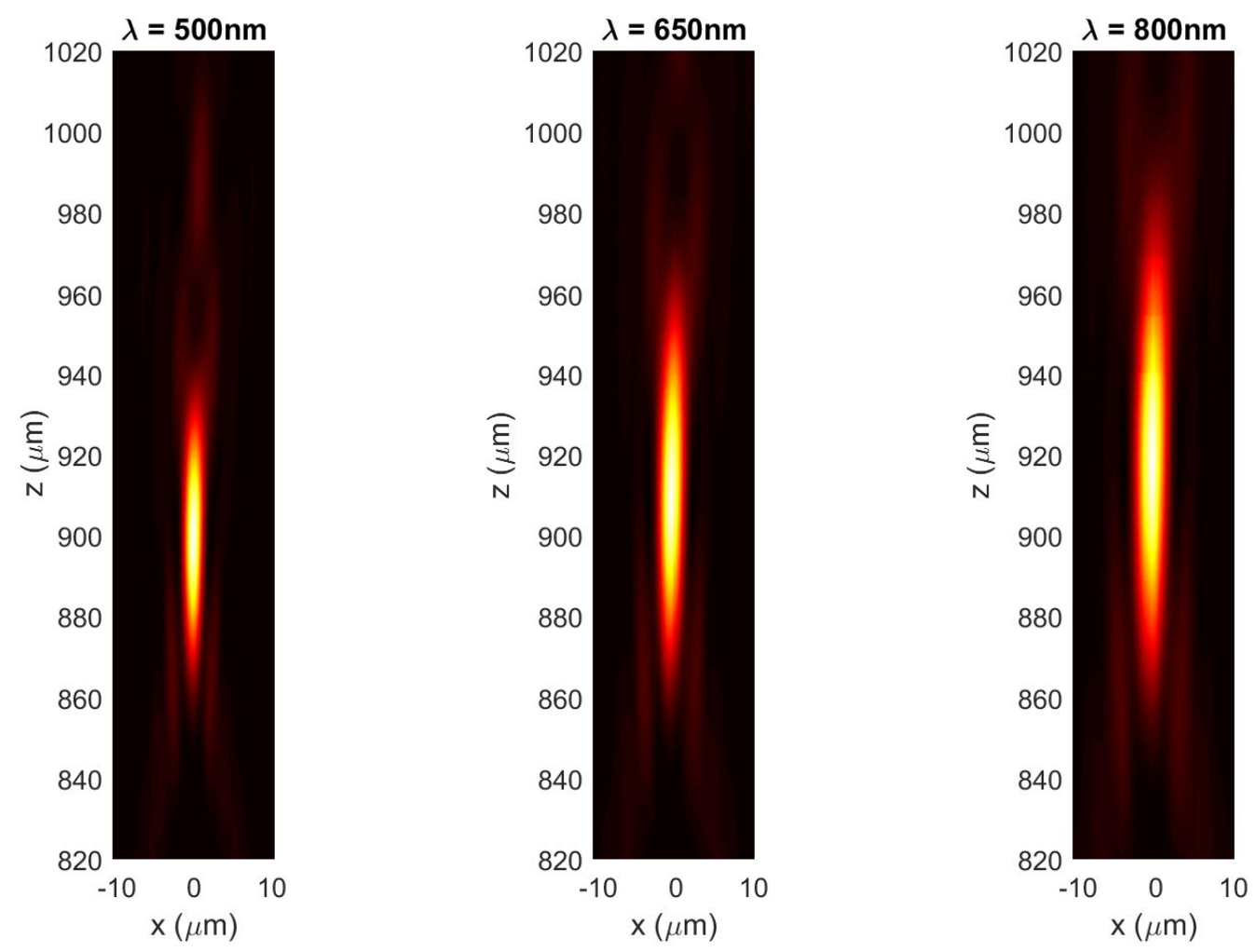

Figure 6: Intensity distribution at the front focal spot for different wavelengths. The origin of the z-axis is set to be the flat outer rim of the microlens.

\subsection{Interferometric measurements}

Interferometric measurements are performed at $200 \mu \mathrm{m}$ below focus in order to get the full phase information on the detector. This measurement provides the wavefront aberration, see Fig. 7a. Its decomposition into standard orthogonal polynomials allows to observe what are the main aberrations of the microlens, Fig. 7b. From this measurement, standard figures of merit such as the Peak-to-Valley or RMS values of wavefront deviation can be calculated.

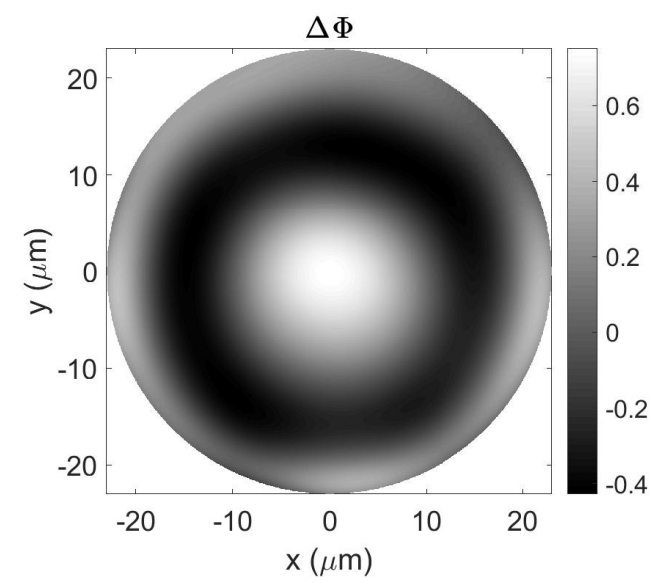

(a)

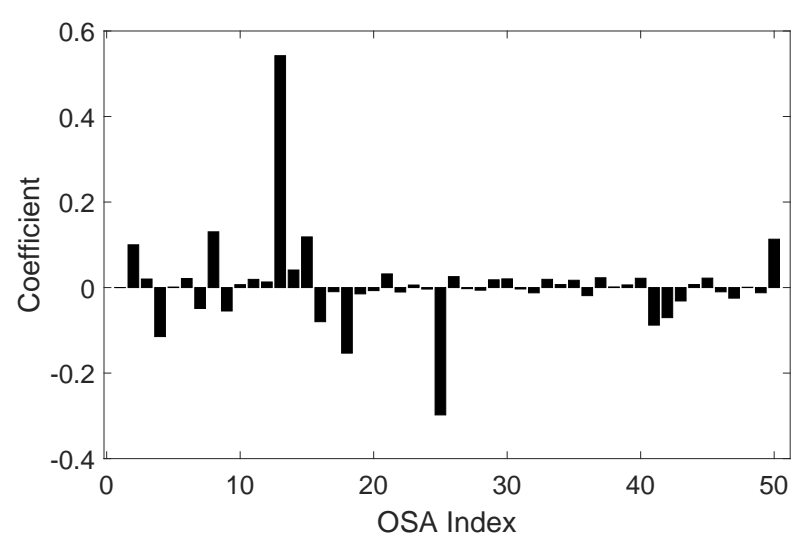

(b)

Figure 7: Phase deviation from spherical wavefront (a) and the corresponding Zernike coefficients (b), $\lambda=600 \mathrm{~nm}$. 
For low aberrated systems, the RMS value of the wavefront deformation is linked to the Strehl ratio by the following approximation [14]

$$
S R \approx e^{-\Delta \Phi_{r m s}^{2}},
$$

which gives a SR of 0.8 for the tested microlens. This result is thus consistent with the SR obtained by irradiance measurement.

For thin microlenses, the phase change is essentially due to the surface height variation and is given by $\Delta \Phi=k n \Delta z$. This allows to reconstruct the microlens surface if the interferometric measurement is performed at the output plane of the microlens. In this case, interferometry is consequently equivalent to profilometry. When the phase is collected between the microlens and the focal spot, linking surface and phase is less straightforward but can nevertheless be performed. To illustrate that, the phase deviation shown in Fig. 7a is compared to the surface deviation given by confocal microscopy, Fig. 8a. The good correlation between the two residuals demonstrates the equivalence of the measurements for process optimization purposes. Indeed, interferometric and profilometric residuals are almost the same, $31.5 \mathrm{~nm}$ vs $32 \mathrm{~nm}$. Small differences are likely due to aberrations of the microscope objectives of the HRIM and of the confocal microscope.

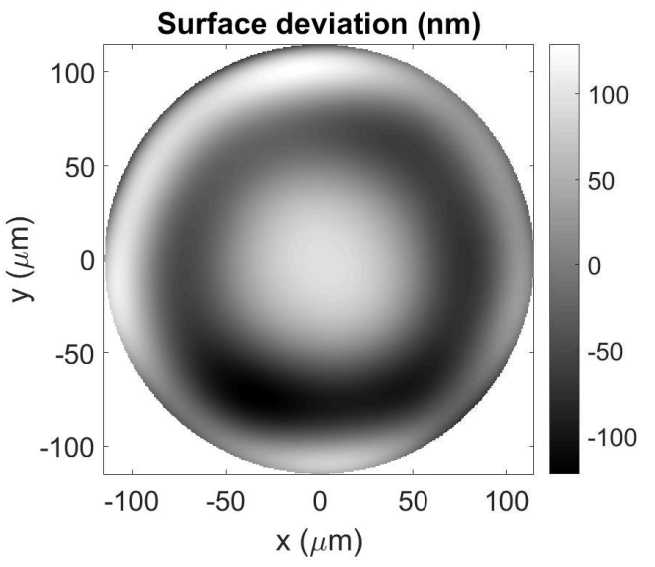

(a)

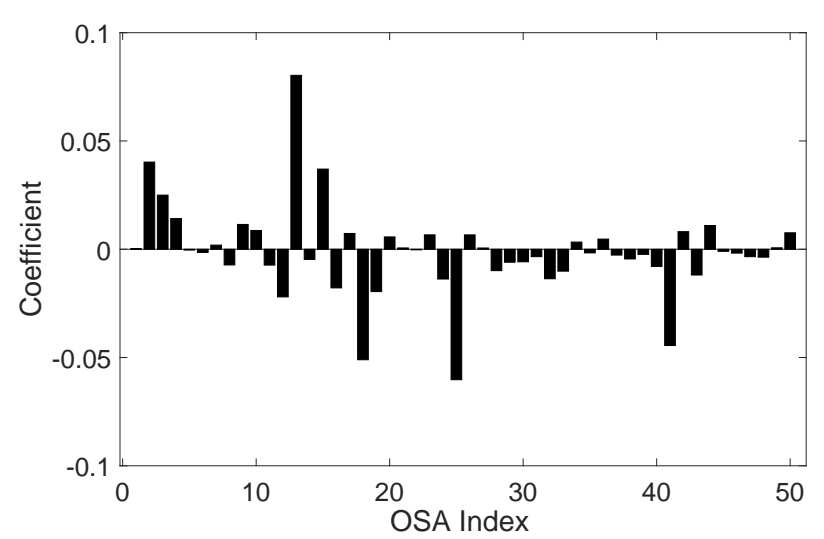

(b)

Figure 8: Surface deviation from the ideal surface (a) and the corresponding Zernike coefficients (b).

\section{CONCLUSION}

The use of a sHRIM to characterize a low NA diffraction limited fused silica microlens is demonstrated. Imaging quality of the microlens is established thanks to interferometric and irradiance measurements at different wavelengths. Surface parameters, such as the ROC and the surface deviation, which are essential for manufacturing process optimization, are also obtained. With this extensive characterization, the use of the sHRIM is thus believed to extend the capacity to fabricate state-of-the-art high NA microlenses and MLAs.

\section{ACKNOWLEDGMENTS}

The authors want to thank A. Vetter and R. Kirner for fruitful discussions.

\section{References}

[1] H. Zappe, Fundamentals of Micro-Optics, Cambridge University Press. Google-Books-ID: OgwksRktpCQC.

[2] P. Nussbaum, R. Völkel, H. P. Herzig, M. Eisner, and S. Haselbeck, "Design, fabrication and testing of microlens arrays for sensors and microsystems," 6(6), p. 617.

[3] T.-K. Shih, C.-F. Chen, J.-R. Ho, and F.-T. Chuang, "Fabrication of PDMS (polydimethylsiloxane) microlens and diffuser using replica molding," 83(11), pp. 2499-2503. 
[4] S. Reichelt and H. Zappe, "Combined twyman-green and mach-zehnder interferometer for microlens testing," 44(27), pp. 5786-5792.

[5] M. Baranski, S. Perrin, N. Passilly, L. Froehly, J. Albero, S. Bargiel, and C. Gorecki, "A simple method for quality evaluation of micro-optical components based on 3d IPSF measurement," 22(11), pp. 13202-13212.

[6] C. Rockstuhl, I. Marki, T. Scharf, M. Salt, H. Peter Herzig, and R. Dandliker, "High resolution interference microscopy: a tool for probing optical waves in the far-field on a nanometric length scale," 2(4), pp. 337-350.

[7] M.-S. Kim, T. Scharf, S. Mühlig, C. Rockstuhl, and H. P. Herzig, "Engineering photonic nanojets," 19(11), pp. 10206-10220.

[8] M.-S. Kim, T. Scharf, S. Mühlig, C. Rockstuhl, and H. P. Herzig, "Gouy phase anomaly in photonic nanojets," 98(19), p. 191114.

[9] M. Born and E. Wolf, Principles of Optics, Chapter VII, Cambridge University Presss, 7th (expanded) ed.

[10] E. P. Goodwin and J. C. Wyant, Field Guide to Interferometric Optical Testing, SPIE Field Guides, SPIE.

[11] P. de Groot, Optical Measurement of Surface Topography, Chapter 8, Springer.

[12] P. Hariharan, B. F. Oreb, and T. Eiju, "Digital phase-shifting interferometry: a simple error-compensating phase calculation algorithm," 26(13), pp. 2504-2506.

[13] D. W. Ghilia and M. D. Pritt, Two-Dimensional Phase Unwrapping Theory, Algorithms and Software, John Wiley \& Sons, Inc.

[14] H. Gross, Handbook of Optical Systems V 3 -Aberration Theory and Correction of Optical Systems, Wiley VCH.

[15] I. H. Malitson, "Interspecimen comparison of the refractive index of fused silica*, $\dagger$ " 55(10), pp. 1205-1209.

[16] Y. Li and E. Wolf, "Focal shift in focused truncated gaussian beams," 42(3), pp. 151-156. 